\title{
Leucine Exerts Lifespan Extension and Improvement in Three Types of Stress Resistance (Thermotolerance, Anti-Oxidation and Anti-UV Irradiation) in C. elegans
}

\author{
Hongyuan Wang ${ }^{1,2}$, Jin Wang ${ }^{2}$, Zhizhou Zhang,2,* \\ ${ }^{1}$ School of Chemistry and Chemical Engineering, Harbin Institute of Technology, Harbin 150001, China \\ ${ }^{2}$ School of Marine Science and Technology, Harbin Institute of Technology, Weihai 264209, China \\ *Corresponding author: zhangzzbiox@hitwh.edu.cn
}

Received September 01, 2018; Revised October 10, 2018; Accepted November 18, 2018

\begin{abstract}
Recent studies have found various compounds that can extend lifespan in different species. Amino acids as regulators in nutrition process and anti-aging have been investigated, but inconsistency existed in the literature in the context of lifespan-extending roles of some amino acids in $C$. elegans. In this paper, we measured the effects of individual branched-chain amino acids (BCAAs, leucine, valine and isoleucine) on lifespan in C. elegans. We found that $1000 \mu \mathrm{M}$ and $10000 \mu \mathrm{M}$ leucine could extend lifespan significantly coupled with increased stress resistance of thermotolerance, anti-oxidation and anti- UV irradiation. Furthermore, we used daf-2 and daf-16 mutants to explore the possible molecular mechanism of Leu-induced lifespan extension. Results suggested that the function of Leu on aging regulation is dependent on Insulin/IGF-1 (IIS) signaling. Our work confirmed that BCAAs play an important role in IIS signaling pathway to regulate aging and intake of such nutrients may also be good for healthspan in C. elegans.
\end{abstract}

Keywords: C. elegans, leucine, aging, stress resistance, daf-16

Cite This Article: Hongyuan Wang, Jin Wang, and Zhizhou Zhang, "Leucine Exerts Lifespan Extension and Improvement in Three Types of Stress Resistance (Thermotolerance, Anti-Oxidation and Anti-UV Irradiation) in C. elegans." Journal of Food and Nutrition Research, vol. 6, no. 10 (2018): 665-673. doi: 10.12691/jfnr-6-10-8.

\section{Introduction}

Aging can be regarded as degeneration of organisms to maintain normal functions in late days, which increases risk of diseases and death. However, the fact that different species have relatively fixed lifespans suggests that lifespan determination shall be a highly regulated process. Thanks to advanced knowledge in aging research, several age-related phenotypes and longevity regulators have been identified. One easy way to study aging is to use simple, experimentally cultured organismal models such as Caenorhabditis elegans (C. elegans). Since C. elegans was widely used as laboratory research organism, a number of compounds and signaling pathways have been demonstrated to regulate lifespan significantly [1]. Regulatory mechanism of aging is complex, coupled by genes and external environment simultaneously. Some papers have discovered classical signaling pathways that could regulate lifespan in short-lived animals including C. elegans and Drosophila and confirmed the similar effects on mammals [2]. For examples, inhibitory of Insullin / IGF-1 signaling and TOR signaling can lead to lifespan extension in various animals, from nematodes to mice [2-10]. On the other side, overexpression of AMP kinase and sirtuins has reported to prolong lifespan $C$.elegans as well $[2,11,12]$. Furthermore, recent aging research also focuses on screening the effect of chemicals and drugs on aging. A series of papers have reported that intake of several compounds, included ethanol, metformin and D-allulose, could extend lifespan significantly in C. elegans through different mechanisms $[13,14,15]$.

Meanwhile, Lopez-Otin et al reported in 2013 that deregulated nutrient-sensing is one important aging- related hallmark [16]. It indicated that intake of necessary nutrition may result in delay in organismal aging. So exploring what are the essential nutritional factors for prolonging lifespan is good to promote aging research. Amino acids are widely known as essential nutrition in our diet. The effect of amino acids on lifespan extension in both C. elegans and Drosophila was confirmed [17,18,19,20]. Recent intriguing researches also indicated that BCAA-enriched mixture (BCAAem) consisting of dominant L-valine, L-isoleucine, and L-leucine plus some other amino acids could increase the average lifespan of mice steadily [21]. This was likely the consequence of increased mitochondrial biogenesis and reduced oxidative stress in cardiac and skeletal muscles via eNOS-mediated mechanisms. The similar longevity-promoting effect of both BCAAem and pure BCAAs on survival rate in Saccharomyces cerevisiae was also reported [22]. However, there is limited information how individual branched-chain amino acid affects the aging rate in C. elegans. 
In this paper, we first did a screening of individual branched-chain amino acid (Val, Leu and Ile) at three different concentrations on the lifespan of the wild type C. elegans. We found only $1000 \mu \mathrm{M}$ and $10000 \mu \mathrm{M}$ Leu could extend lifespan significantly. Then we observed how Leu supplementation change physiological characters of nematodes and checked if Leu could promote resistance to stress included heat shock, oxidative stress and UV irradiation. Results showed that lifespan extension in Leu-treated worms was accompanied with improved resistance to thermal treatment, oxidative stress and UV irradiation. Then we confirmed Leu-induced lifespan extension was dependent on daf-2 and daf-16 which were two important genes in IIS signaling pathway. We also detected the raised mRNA expression of daf-16 using quantitative real-time PCR. In summary, our results suggested supplementary Leu could extend lifespan through increased stress resistance and inhibited IIS signaling.

\section{Material and Methods}

\subsection{Chemicals, Strains and Culture}

L-Valine (Val), L-Leucine (Leu) and L-Isoleucine (Ile) were purchased from Sangon Biotech (Shanghai, China) and dissolved in sterile $\mathrm{ddH}_{2} \mathrm{O}$. Worms were cultured at $20^{\circ} \mathrm{C}$ as previously described unless otherwise stated [23]. Strains used in this paper were N2 CGCH [24], CB1370 daf-2(e1370) III, and CF1038 daf-16(mu86) I. All strains were provided by Caenorhabditis Genetics Center (CGC).

\subsection{Lifespan Assays}

Several young adult worms were cultured on nematode growth medium (NGM) seeded with E. coli OP50 for laying eggs. One day later plates with gravid adults and eggs were used for synchronization to get plenty healthy eggs. All the eggs were cultured on NGM plates and NGM plates added with different concentrations of BCAAs separately. Two days later L4 larvae were transferred to different kinds of NGM plates. The day of L4 stage was recorded as day 0 and survival rate was counted every day until all the worms were dead.

\subsection{Brood Size Counting}

Single worm was cultured on NGM plates with or without Leucine from L4 stage. There were 15 plates for each group. Worms were transferred to new plates every 24 hours and eggs in the old plates were counted.

\subsection{Body Length Measurement}

Random 3 worms per group were treated with $0.2 \%$ levamisole from day 5 to day 10 . Yongheng $\mathrm{XYH}-3 \mathrm{~A}$ microscope was used to take pictures of worms and Shangguang Camera software was used to measure body length.

\subsection{Stress Resistance Assays}

Synchronized larvae were put on NGM plates with or without Leucine and cultured at $20^{\circ} \mathrm{C}$ for 5 days. All the stress resistance experiments were repeated three times (90 worms per group in total) as previously described [25]. For Heat shock experiment, 30 worms per group were transferred to $40^{\circ} \mathrm{C}$ incubator. Survival rate was counted every hour after heat shock. For Oxidative stress experiment, $1.5 \mathrm{~g} / \mathrm{L}$ paraquat was added to each plate on day 5 after synchronization to induce oxidative damage. Survival rate was counted every hour. For UV irradiation experiment, 30 worms were transferred to Leu treated NGM plates at L4 stage and UV-irradiated with the use of a JS-350B handheld Ultraviolet Lamp (Peiqing, Shanghai) equipped with two 254nm bulbs (15w per bulb) from day 5 after synchronization. The handheld Ultraviolet Lamp was put about $12 \mathrm{~cm}$ away from NGM plates with nematodes. After UV irradiation survival rate was counted every 12 hours.

\subsection{Gene Expression}

Synchronized worms were put onto NGM plates added with $1000 \mu \mathrm{M}$ at $20^{\circ} \mathrm{C}$ for 10 days. Total RNA was extracted from nematodes using UNIQ-10 column Trizol total RNA extraction kit (Sangon, Shanghai) and was reverse transcribed into cDNA using AMV first strand cDNA synthesis kit (Sangon, Shanghai). The expression of mRNA was detected by quantitative real-time PCR on Applied Biosystems Step-one system. Gene expression data was analyzed using the comparative $2^{-\Delta \Delta \mathrm{Ct}}$ method with GAPDH as the reference gene. The sequences of primers used in this paper were as follows: GAPDH, 5'-TCG CCA AGG AAG GAA AGT-3' (F) and 5'-AAG TGG AGC AAG GCA GTT AG-3' (R). DAF-16, 5'-ATG ATG GAG CCT TAC TTG GA-3' (F) and 5'-CTT GTG GAT TTG CAT TTG TG-3' (R).

\subsection{Statistical Analysis}

GraphPad Prism 6.02 was used to perform data analysis. Kaplan-Meier survival analysis and log-rank were used to analyze survival rate. Multiple comparison t test was used to compare body length and brood size.

\section{Results}

\subsection{Leucine Extends Lifespan in Wild-Type C. elegans}

We checked the effect of BCAAs on lifespan in wild-type $C$. elegans at different concentrations. From the results we found supplementary L-Valine (Val) could shorten lifespan significantly at all concentrations. However, worms fed with more than $1000 \mu \mathrm{M}$ L-Leucine (Leu) lived longer than those cultured on normal NGM plates. Compared to the control group it showed $11.4 \%$ $(\mathrm{p}<0.05)$ and $13.1 \%(\mathrm{p}<0.01)$ lifespan extension caused by supplementation of $1000 \mu \mathrm{M}$ and $10000 \mu \mathrm{M}$ Leu separately (Figure 1 and Table 1). 
A<smiles>CC(C)C[C@H](N)C(=O)O</smiles>

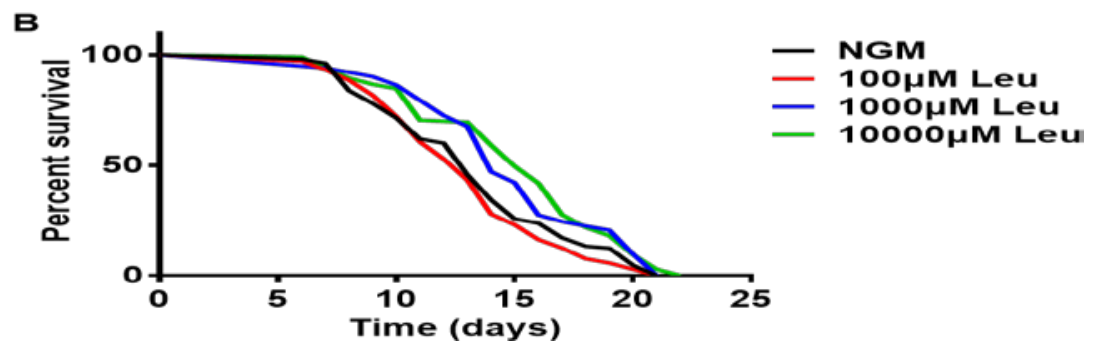

Figure 1. Leucine extends lifespan significantly in C. elegans. (A) Chemical structure of L-Leucine. (B) Survival curves of wild-type nematodes treated with 0 (black), $100 \mu \mathrm{M}$ (red), $1000 \mu \mathrm{M}$ (blue) and $10000 \mu \mathrm{M}$ (green) Leu

Table 1 BCAAs have differential effects on lifespan of C.elegans

\begin{tabular}{|c|c|c|c|c|c|c|c|}
\hline Genotype & Amino acids & Number of worms & Number of trials & Median lifespan & Mean lifespan ( \pm SEM) & $P$ value & Change (\%) \\
\hline \multirow{12}{*}{ N2 } & 0 & 104 & 3 & 13 & $13.54 \pm 0.3429$ & & \\
\hline & $100 \mu \mathrm{M} \mathrm{Val}$ & 104 & 3 & 12 & $11.37 \pm 0.2915$ & $<0.0001^{* * * *}$ & $-16.1 \%$ \\
\hline & $1000 \mu \mathrm{M} \mathrm{Val}$ & 104 & 3 & 12 & $12.54 \pm 0.3031$ & $0.0097 * *$ & $-7.4 \%$ \\
\hline & $10000 \mu \mathrm{M} \mathrm{Val}$ & 99 & 3 & 9 & $9.475 \pm 0.2387$ & $<0.0001^{* * * *}$ & $-30.0 \%$ \\
\hline & 0 & 105 & 3 & 13 & $13.27 \pm 0.3962$ & & \\
\hline & $100 \mu \mathrm{M}$ Leu & 104 & 3 & 13 & $12.86 \pm 0.3577$ & 0.2695 & $-3.1 \%$ \\
\hline & $1000 \mu \mathrm{M}$ Leu & 102 & 3 & 14 & $14.78 \pm 0.3880$ & $0.0119 *$ & $+11.4 \%$ \\
\hline & $10000 \mu \mathrm{M}$ Leu & 105 & 3 & 15 & $15.00 \pm 0.4053$ & $0.0014^{* *}$ & $+13.1 \%$ \\
\hline & 0 & 104 & 3 & 13 & $13.25 \pm 0.3012$ & & \\
\hline & $100 \mu \mathrm{M}$ Ile & 104 & 3 & 13 & $13.43 \pm 0.1965$ & 0.3094 & $+1.4 \%$ \\
\hline & $1000 \mu \mathrm{M}$ Ile & 103 & 3 & 15 & $14.02 \pm 0.2861$ & 0.2035 & $+5.8 \%$ \\
\hline & $10000 \mu \mathrm{M}$ Ile & 102 & 3 & 14 & $13.72 \pm 0.2054$ & 0.5556 & $+3.5 \%$ \\
\hline \multirow{2}{*}{ daf-2 } & 0 & 90 & 3 & 25 & $23.71 \pm 0.8606$ & & \\
\hline & $1000 \mu \mathrm{M}$ Leu & 90 & 3 & 25 & $23.29 \pm 0.8683$ & 0.6952 & $-1.8 \%$ \\
\hline \multirow{2}{*}{ daf-16 } & 0 & 90 & 3 & 9 & $9.756 \pm 0.3232$ & & \\
\hline & $1000 \mu \mathrm{M}$ Leu & 90 & 3 & 9 & $9.089 \pm 0.3190$ & 0.1408 & $-6.8 \%$ \\
\hline
\end{tabular}

$\mathbf{A}$
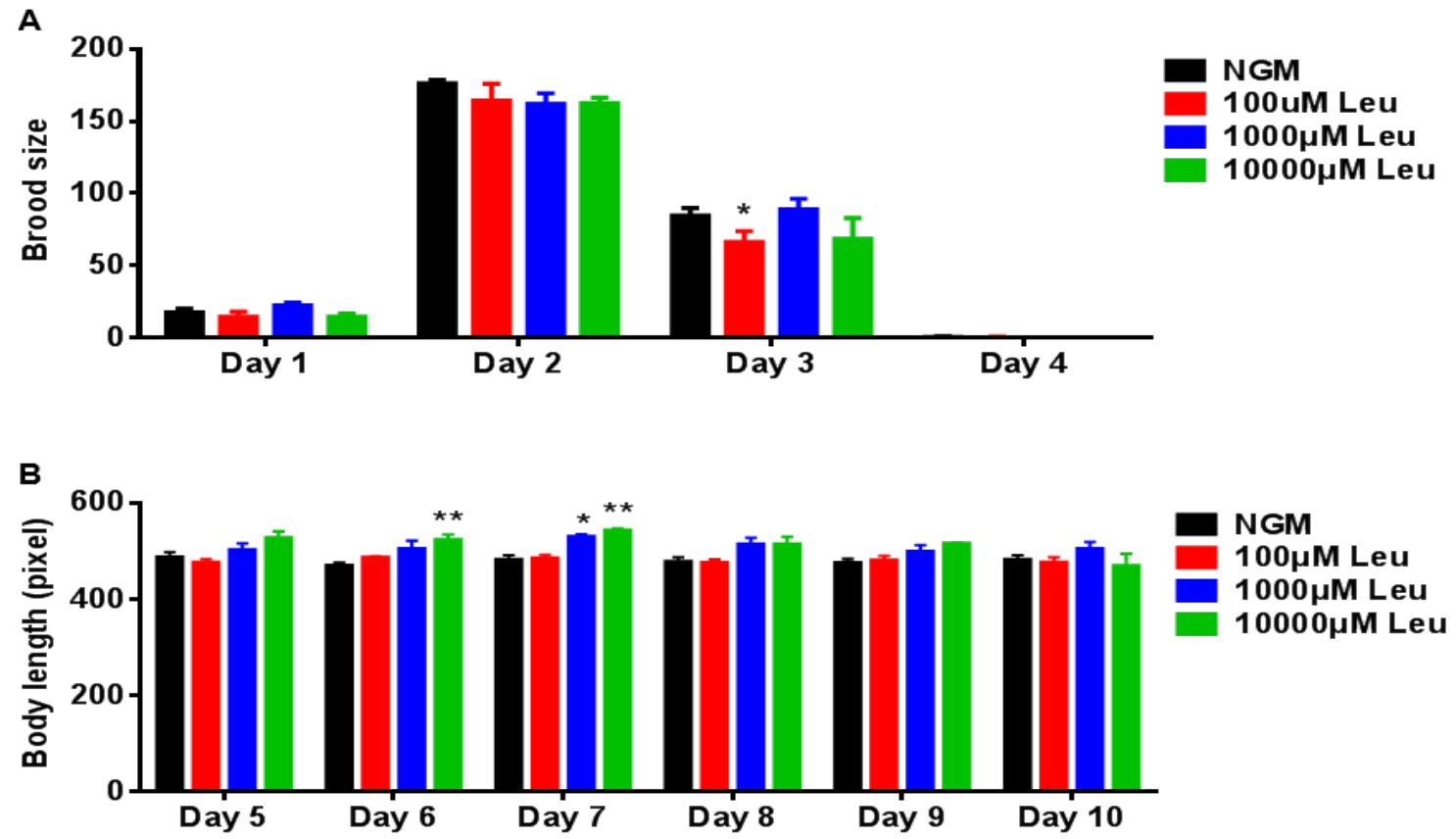

Figure 2. Leu-treated worms exert changes in body length and brood size. (A) Body length of nematodes treated with 0 (black), $100 \mu \mathrm{M}$ (red), $1000 \mu \mathrm{M}$

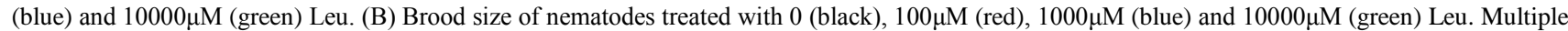
t-test was used for both experiments. ${ }^{*} p<0.05,{ }^{* *} p<0.01$. 


\subsection{Leucine Increases Body Length Slightly}

We observed changes of worms caused by added Leu by measuring body length from day 5 to day 10 after L4 stage and counting brood size. The conclusion was that lifespan extension induced by high concentration Leu was not coupled with change of brood size (Figure 2A). Interestingly, both $1000 \mu \mathrm{M}$ and $10000 \mu \mathrm{M}$ Leu could increase body length significantly on day 7 and $10000 \mu \mathrm{M}$ Leu even caused longer bodies of worms on day 6 as well (Figure 2B). It suggested that $1000 \mu \mathrm{M}$ Leu and $10000 \mu \mathrm{M}$ Leu could extend $C$. elegans lifespan without changing reproductive ability and delaying development obviously.

\subsection{Leucine Improves Stress Resistance}

Several experiments were performed to detect whether supplemental Leu could improve resistance in C. elegans to strict stresses including heat shock, oxidative damage and UV irradiation. Survival rate and mobility analysis were used as indicators to determine healthspan of worms. Results suggested that Leu-treated worms show significantly improved resistance to high temperature plus more significantly improved resistance to added paraquat and UV exposure (Figure 3). The survival rate of worms treated with $1000 \mu \mathrm{M}$ and $10000 \mu \mathrm{M}$ Leu was significantly higher than untreated worms when faced with oxidative pressure by paraquat. Additionally there were 2-3 fold more active worms after 2 hours fed with paraquat in Leu treated groups (Figure 3B). It is interesting that Leu-treated worms also showed improved resistance on both extended lifespan and increased proportion of active worms under UV irradiation condition (Figure 3C).

\subsection{Lifespan Extension Induced by Leu is Dependent on IIS Signaling Pathway}

Insulin / IGF-1 signaling pathway has been proved to be one of the most important pathways in regulating longevity and is conserved in various organisms. To further explore the possible molecular mechanisms of longevity regulation we also observed the effect of $1000 \mu \mathrm{M}$ Leu on lifespan in daf-2 and daf-16 mutants. Interestingly, although Leu-treated wild-type worms lived longer than untreated worms, the lifespan of daf-2 and daf-16 mutants treated with $1000 \mu \mathrm{M}$ Leu was similar to the control group (Figure 4A, B and Table 1). It indicated that lifespan extension caused by Leu supplementation was dependent on IIS signaling pathway. The mRNA expression of DAF16 was analyzed using quantitative real-time PCR. Results showed that $1000 \mu \mathrm{M}$ Leu increased expression of DAF-16 in wild-type worms significantly (Figure $4 \mathrm{C}$ ).
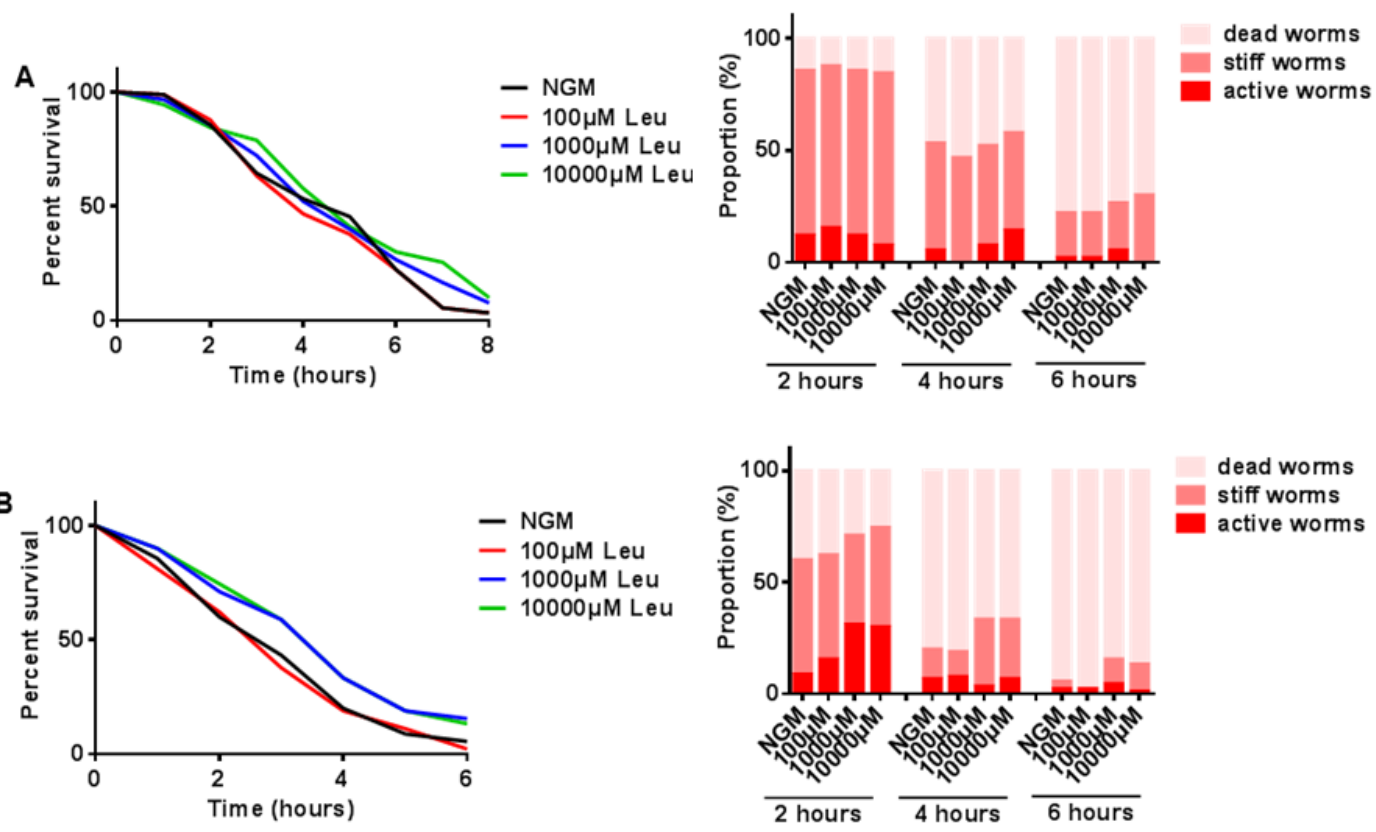

C

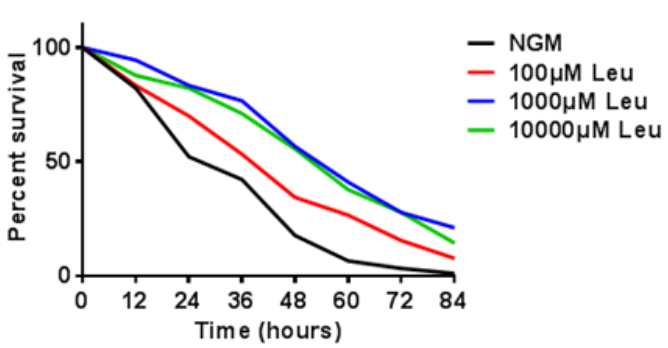

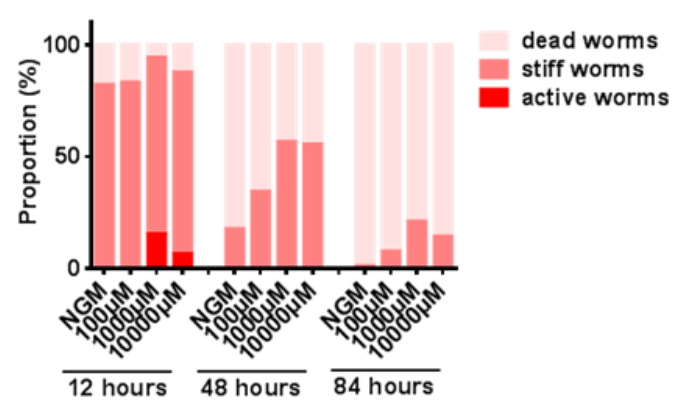

Figure 3. Leu increased stress resistance in C. elegans. (A) Survival curve (left) and proportion analysis of average motility (right) of nematodes treated with 0 (black), $100 \mu \mathrm{M}$ (red), $1000 \mu \mathrm{M}$ (blue) and $10000 \mu \mathrm{M}$ (green) Leu under heat shock condition. (B) Survival curve (left) and proportion analysis of average motility (right) of nematodes treated with 0 (black), $100 \mu \mathrm{M}$ (red), $1000 \mu \mathrm{M}$ (blue) and $10000 \mu \mathrm{M}$ (green) Leu under paraquat in NGM condition. (C) Survival curve (left) and proportion analysis of average motility (right) of nematodes treated with 0 (black), $100 \mu \mathrm{M}$ (red), $1000 \mu \mathrm{M}$ (blue) and $10000 \mu \mathrm{M}$ (green) Leu under UV irradiation condition 

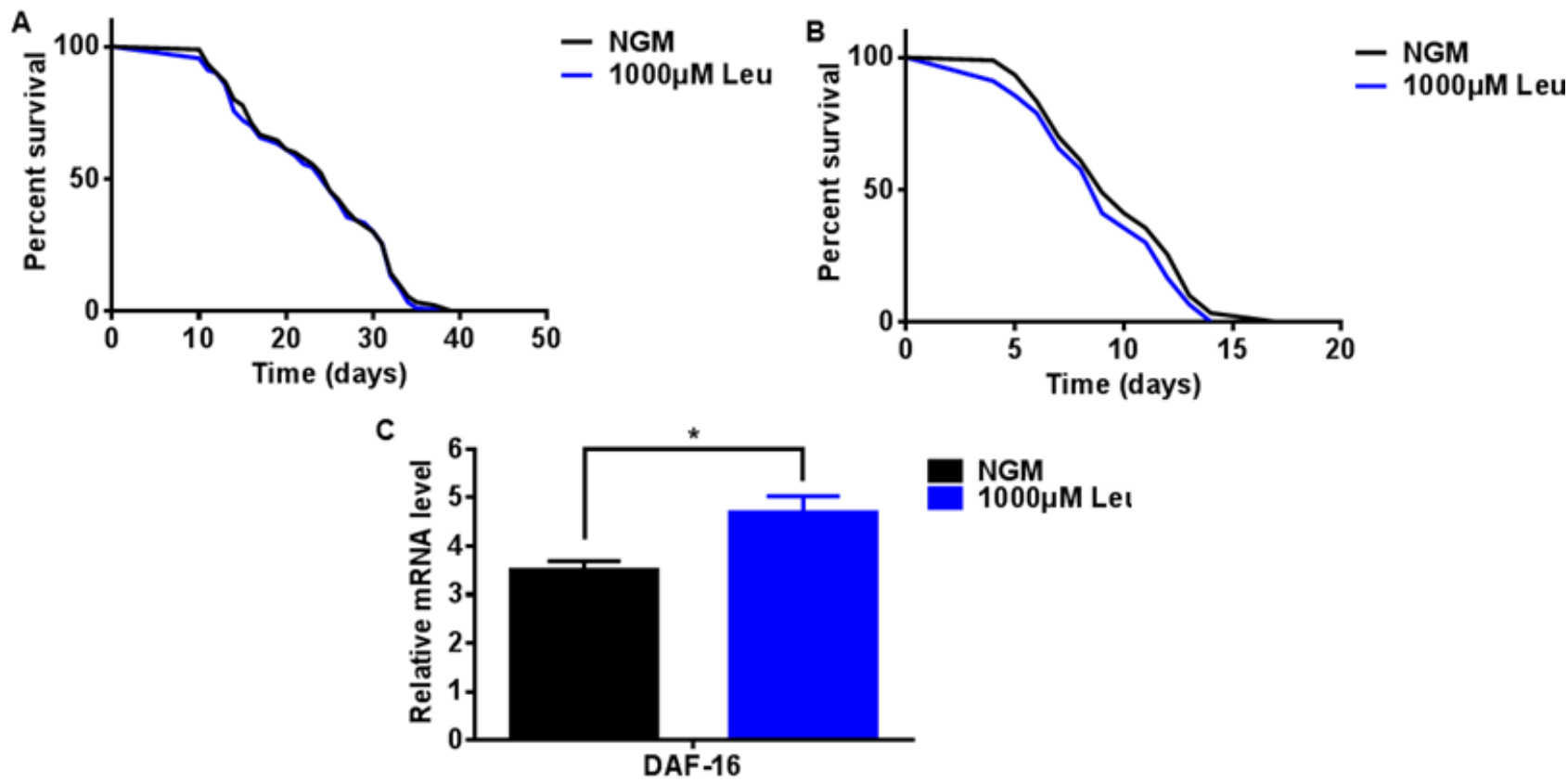

Figure 4. Lifespan extension induced by supplementary Leu is dependent on IIS signaling pathway. (A) Survival curve of daf-2 mutant of nematodes treated with 0 (black) and $1000 \mu \mathrm{M}$ (blue) Leu. (B) Survival curve of daf-16 mutant of nematodes treated with 0 (black) and $1000 \mu \mathrm{M}$ (blue) Leu. (C) mRNA expression of DAF-16 analyzed by quantitative real-time PCR of worms treated with 0 (black) and $1000 \mu \mathrm{M}$ (blue) Leu. ${ }^{*} p<0.05$.

Table 2. Amino acids other than BCAAs have differential effects on lifespan of $C$ elegans

\begin{tabular}{|c|c|c|c|c|}
\hline & Amino acids & Effects on lifespan & $P$ value & Reported results under similar conditions [17] \\
\hline 1 & 10 Cys & - & $\mathrm{p}=0.003^{* *}$ & + \\
\hline 2 & 10 Asp & - & $\mathrm{p}=0.026^{*}$ & $\mathrm{n}$ \\
\hline 3 & 10 Asn & - & $\mathrm{p}=0.001^{* *}$ & + \\
\hline 4 & $1 \mathrm{Tyr}$ & - & $\mathrm{p}=0.026^{*}$ & + \\
\hline 5 & 0.1 Try & - & $\mathrm{p}=0.012 *$ & \\
\hline 6 & 10 Try & - & $\mathrm{p}=0.034^{*}$ & + \\
\hline 7 & $1 \mathrm{Thr}$ & - & $\mathrm{p} \approx 0.000 * *$ & $\mathrm{n}$ \\
\hline 8 & $10 \mathrm{Thr}$ & - & $\mathrm{p}=0.001^{* *}$ & $\mathrm{n}$ \\
\hline 9 & 1 Lys & - & $\mathrm{p}=0.046^{*}$ & + \\
\hline 10 & 0.1 Ala & + & $\mathrm{p}=0.009 * *$ & \\
\hline 11 & 0.1 His & + & $\mathrm{p}=0.018^{*}$ & \\
\hline 12 & $1 \mathrm{His}$ & + & $\mathrm{p}=0.027^{*}$ & $\mathrm{n}$ \\
\hline 13 & 1 Met & + & $\mathrm{p}=0.011^{*}$ & $\mathrm{n}$ \\
\hline 14 & $10 \mathrm{Met}$ & + & $\mathrm{p}=0.001 * *$ & + \\
\hline 15 & 0.1 Pro & + & $\mathrm{p}=0.035^{*}$ & \\
\hline
\end{tabular}

Note: 28-35 worms were used for each experiment.- and+ represent significant $\left({ }^{*} p<0.05\right.$ or $\left.{ }^{* *} p<0.01\right)$ attenuation and elongation of lifespan, respectively. The letter $\mathrm{n}$ means no effect. Each amino acid has three concentrations $(0.1 \times, 1 \times$ and $10 \times)$.All amino acids in this table were added into 100 $\mathrm{mL}$ NGM medium by $0.0076 \mathrm{~g}$ for the concentration of $1 \times$ (around $0.5 \mathrm{mM}$ or $500 \mu \mathrm{M}$ for each amino acid).See Wang Hongyuan thesis for Master degree, 2013, Harbin Institute of Technology (Weihai).

\section{Discussion}

Previous research [17] reported that serine and proline, not leucine, showed the largest effects on lifespan extension for C. elegans at the similar experimental conditions as in our lab. Our lab has also scanned amino acids other than BCAAs for their effects on lifespan in C. elegans using the N2 strain and the similar experimental conditions [Wang Hongyuan thesis for Master degree, 2013, Harbin Institute of Technology (Weihai)], and found that around half amino acids shortened the lifespan (Table 2), different from the literature [17] that most of the 20 tested amino acids extended the lifespan. One of the probable reasons for this discrepancy may lie on the fact that scanning of 20 amino acids needs a large number of worms to manipulate by hand and is very liable to bring experimental errors, while the worm number of around 30 is not enough for accurate lifespan determination. Detailed data can be found in Table S1. After three batches of experiments, with each experiment using 28-35 worms, Cys (10×), Asn $(10 \times)$, Thr $(10 \times)$, Asp $(10 \times)$, Tyr $(1 \times)$, and Met $(1 \times)$ were very stable for their effects on the lifespan (Table S2). 
Table 3. Amino acids promotes longevity and certain biological activities through differential molecular mechanisms

\begin{tabular}{|c|c|c|c|}
\hline & Phenotypes/Biological functions & Pathways/Key genes & Organism \\
\hline 1 & $\begin{array}{l}\text { Doubling dietary Leu reversed many of the metabolite abnormalities and caused } \\
\text { a marked improvement in glucose tolerance and insulin signaling without } \\
\text { altering food intake or weight gain [30] }\end{array}$ & insulin signaling, mTOR & mice \\
\hline 2 & $\begin{array}{l}\text { Elevated Leu, Val and Ile plus other amino acid mixture increased the average } \\
\text { lifespan with mitochondria biogenesis [21] }\end{array}$ & $\begin{array}{l}\text { eNOS-mediated pathway; } \\
\text { mTOR signaling, }\end{array}$ & middle-aged mice \\
\hline 3 & $\begin{array}{l}\text { Muscle protein synthesis was stimulated by Leu-rich whey not by Leu alone } \\
\text { [31] }\end{array}$ & Akt & Aged mice \\
\hline 4 & $\begin{array}{l}\text { Under nitrogen fertilization, Leu and Met accumulated in green morph enhanced } \\
\text { TOR signaling, but not in red morph [32] }\end{array}$ & TOR & Morph \\
\hline 5 & Leu and Ile independently regulate mTORC1 activity and protein synthesis [28] & mTOR & $\begin{array}{l}\text { Human cell, bovine } \\
\text { mammary tissue }\end{array}$ \\
\hline 6 & $\begin{array}{l}\text { Mitochondrial respiration or ubiquinone production is inhibited; may influenced } \\
\text { by Leu and Ile [33] }\end{array}$ & Clk-1; fstr-1/2 signaling & C. elegans \\
\hline 7 & Leu induces mitochondrial biogenesis and oxidative function [34] & AMPK/Sirt1 signaling & C2Cl2 myotubes \\
\hline 8 & $\begin{array}{l}\text { AMPK and Sirts modulate observed effects of metformin with Leu in muscle, } \\
\text { liver and adopose tissues [35] }\end{array}$ & AMPK/Sirt1 signaling & mice \\
\hline 9 & Leu extends lifespan and improves tolerance to oxidative stress [27] & AMPK, Sir2.1, & C. elegans \\
\hline 10 & $\begin{array}{l}\text { Leu extends lifespan and improves thermotolerance and tolerance to oxidative } \\
\text { stress }\end{array}$ & DAF2, DAF16 & C. elegans \\
\hline 11 & $\begin{array}{l}\text { Sulfur amino acid restriction increased capillary density in mouse skeletal } \\
\text { muscle in vivo [36] }\end{array}$ & $\begin{array}{l}\text { GCN2/ATF4 amino acid } \\
\text { startvation response pathway }\end{array}$ & mouse \\
\hline 12 & Leu can regulate gene transcription in mammalian tissues [37] & GCN2/eIF2a/ATF4 pathway & mice \\
\hline 13 & $\begin{array}{l}\text { Leu activates the TORC1 kinase via both EGOC GTPase-dependent and - } \\
\text { independent mechanisms [38] }\end{array}$ & TCA cycle; TOR signaling & yeast \\
\hline 14 & $\begin{array}{l}\text { Serine and proline show largest effects on lifespan promotion; Proline and } \\
\text { tryptophan increase thermotolerance; tryptophan-mediated lifespan extension is } \\
\text { independent on DAF-16 [17] }\end{array}$ & $\begin{array}{l}\text { Mitochondrial TCA cycle; } \\
\text { DAF-16/FOXO; SKN-1/Nrt2 } \\
\text { stress response; AMPK } \\
\text { pathway }\end{array}$ & C. elegans \\
\hline 15 & $\begin{array}{l}\text { Met and Leu activate TOR signaling in yeast,while Leu and Arg activate mTOR } \\
\text { signaling in mammals [39] }\end{array}$ & TORC1 & $\begin{array}{l}\text { Yeast } \\
\text { Mammals }\end{array}$ \\
\hline 16 & $\begin{array}{l}\text { Isoamyl alcohol odor promotes longevity and stress tolerance via DAF-16 in } \\
\text { Caenorhabditis elegans [40] }\end{array}$ & DAF-16 & C. elegans \\
\hline
\end{tabular}

Among the 20 amino acids, however, Leu is a specific one that has been heavily studied in the context of lifespan extension, mitochondrial biogenesis and related biological functions in different species, tissues or cell types. Table 3 summarized over 15 reported typical cases in which amino acids, especially Leu, when properly elevated for their concentration levels, prolonged the lifespan of model organisms or induced biological phenotypes that may benefit the lifespan extension. It is clear that Leu is able to work as a regulator for these signaling pathways. But how these pathways coordinate quantitatively under the Leu stimuli needs further investigation in many different cells or tissues. Leucine exerts similar molecular effects on similar signaling pathways in different species, tissues or cell types, but the resulting phenotypes in different species may be pleiotropic. Moreover, yeast genetics studies indicated that lifespan elongation can be achieved in many ways [26], suggesting that lifespan extension is merely a phenotype governed by several interwining pathways and the status of these pathways can be disturbed by many gene mutations, metabolites, nutrients or environmental signals. This is consistent with a dozen of case studies in which Leu can synergistically work with other small molecules to extend lifespan or promote related phenotypes [27].
There are several lines of evidence that Leu is different from Val and Ile in regulation of cellular processes [21, 22, 28], especially, three BCAAs differentially play their own distinct roles for influencing activities of the following signaling pathways: insulin signaling, TOR signaling, mitochondrial biogenesis, AMPK/Sir2.1 signaling, TCA cycle regulation, and stress responding pathways. That is also the reason why this study's first aim was to get clear if all of the three amino acids similarly affect the lifespan of C. elegans.

Previous reports also found that the thermotolerance (under $35^{\circ} \mathrm{C}$ ) of $C$. elegans was improved by amino acid supplementation of proline or tryptophan, but not Leu [17]. Because of the above mentioned discrepancy between literature [17] and our own results, three different types of stresses were tested on Leu-fed worms. In this study, $1000 \mu \mathrm{M}$ Leu supplementation preserved at least 2-fold more active worms under $40^{\circ} \mathrm{C}$ for 6 hrs than the control group (Figure 2A). In the literature [17], amino acids were also tested for the worm's resistance to oxidative stress, but only histidine showed slight protection effect and histidine was not one of those extending the lifespan to the greatest extent. To note, increased UV irradiation protection effect exerted by Leu supplementation was first reported for $\mathrm{C}$. elegans in this study, and the significant 
improvement of survival rate was also observed at $1000 \mu \mathrm{M}$ Leu (Figure 2C). Considering that there is no decreased body length and brood size compared to worms fed with normal E. coli OP50, all these results suggested that supplementary individual BCAA could regulate aging process independent on changing the general developmental process of worms. Plus, added Leu in diet is also beneficial to increase stress resistance, which was also shown in other literature [29]. So intake of Leu may be good for both lifespan and healthspan of the model organism.

In conclusion, in this paper we found that Leu, Val and Ile played different roles for lifespan extension of C. elegans, described the positive effect of Leu supplementation on anti-aging in $C$. elegans and explored the possible molecular mechanism. Especially, the lifespan extension in Leu-treated worms was accompanied with improved resistance to thermal treatment, oxidative stress and UV irradiation. It requires further investigations in the level of systems biology to understand comprehensively how leucine perturbs and orchestrates the metabolic network and signaling pathways. Meanwhile, this work provided more evidence to confirm the benefit of Leu as a possible health- promoting reagent.

\section{Acknowledgements}

C. elegans strains used in this paper were purchased from Caenorhabditis Genetics Center, which is funded by National Institute of Health-Office of Research Infrastructure Programs (P40 OD010440). This study was supported by the following funds: NSFC (No.31071170); GujingTribute fund (2016-1); GREDBIO (201401); the Key research and development plan of Shandong Province (2016GSF115022); 2018 Weihai Scientific Innovation Project, and the Natural Science Foundation of Shandong Province (ZR2018MC002).

\section{Author Contributions}

W.H. and Z.Z. designed research; W.H. and W.J. conducted research; W.H. and Z.Z. wrote the paper. W.H. and Z.Z. had primary responsibility for the final content. All authors read and approved the final manuscript.

\section{Conflict of Interest Statement}

The authors declare that there are no conflicts of interest.

\section{References}

[1] H. Gershon, D. Gershon, "Caenorhabditis elegans--a paradigm for aging research: advantages and limitations", Mechanisms of Ageing and Development, 123 (4). 261-274. February 2002.

[2] C.J. Kenyon, "The genetics of ageing", Nature, 464 (7288). 504- 512. March 2010.

[3] O. Altintas, S. Park, S.J. Lee, "The role of insulin/IGF-1 signaling in the longevity of model invertebrates, C. elegans and D. melanogaster", BMB Reports, 49 (2). 81-92. February 2016.
[4] H. Antikainen, M. Driscoll, G. Haspel, R. Dobrowolski, "TOR-mediated regulation of metabolism in aging", Aging Cell, 16(6). 1219-1233. December 2017.

[5] M. Kaeberlein, R.W. Powers, 3rd, K.K. Steffen, E.A. Westman, D. Hu, N. Dang, E.O. Kerr, K.T. Kirkland, S. Fields, B.K. Kennedy, "Regulation of yeast replicative life span by TOR and Sch9 in response to nutrients", Science, 310 (5751). 1193-1196. November 2005.

[6] P. Kapahi, B.M. Zid, T. Harper, D. Koslover, V. Sapin, S. Benzer, "Regulation of lifespan in Drosophila by modulation of genes in the TOR signaling pathway", Current Biology, 14 (10). 885-890. May 2004.

[7] L. Kappeler, C. De Magalhaes Filho, J. Dupont, P. Leneuve, P. Cervera, L. Perin, C. Loudes, A. Blaise, R. Klein, J. Epelbaum, Y. Le Bouc, M. Holzenberger, "Brain IGF-1 receptors control mammalian growth and lifespan through a neuroendocrine mechanism", PLoS Biology, 6 (10). e254. October 2008.

[8] R. Yuan, S.W. Tsaih, S.B. Petkova, C. Marin de Evsikova, S. Xing, M.A. Marion, M.A. Bogue, K.D. Mills, L.L. Peters, C.J. Bult, C.J. Rosen, J.P. Sundberg, D.E. Harrison, G.A. Churchill, B. Paigen, "Aging in inbred strains of mice: study design and interim report on median lifespans and circulating IGF1 levels", Aging Cell, 8 (3). 277-287. June 2009.

[9] C. Kenyon, "The plasticity of aging: insights from long-lived mutants", Cell, 120 (4). 449-460. February 2005

[10] W.R. Swindell, "Rapamycin in mice", Aging (Albany NY), 9 (9). 1941-1942. September 2017.

[11] J. Apfeld, G. O'Connor, T. McDonagh, P.S. DiStefano, R. Curtis, "The AMP-activated protein kinase AAK-2 links energy levels and insulin-like signals to lifespan in C. elegans", Genes \& Development, 18 (24). 3004-3009. December 2004.

[12] A. Berdichevsky, M. Viswanathan, H.R. Horvitz, L. Guarente, "C. elegans SIR-2.1 interacts with 14-3-3 proteins to activate DAF-16 and extend life span", Cell, 125 (6). 1165-1177. June 2006.

[13] F. Cabreiro, C. Au, K.Y. Leung, N. Vergara-Irigaray, H.M. Cocheme, T. Noori, D. Weinkove, E. Schuster, N.D. Greene, D. Gems, "Metformin retards aging in C. elegans by altering microbial folate and methionine metabolism", Cell, 153 (1). 228-239. March 2013.

[14] P.V. Castro, S. Khare, B.D. Young, S.G. Clarke, "Caenorhabditis elegans battling starvation stress: low levels of ethanol prolong lifespan in L1 larvae", PLoS One, 7(1). e29984. January 2012.

[15] T. Shintani, H. Sakoguchi, A. Yoshihara, K. Izumori, M. Sato, "D-Allulose, a stereoisomer of d-fructose, extends Caenorhabditis elegans lifespan through a dietary restriction mechanism: A new candidate dietary restriction mimetic", Biochemical and Biophysical Research Communications, 493(4). 1528-1533. December 2017.

[16] Lopez-Otin, M.A. Blasco, L. Partridge, M. Serrano, G. Kroemer, "The hallmarks of aging", Cell, 153 (6). 1194-1217. June 2013.

[17] C. Edwards, J. Canfield, N. Copes, A. Brito, M. Rehan, D. Lipps, J. Brunquell, S.D. Westerheide, P.C. Bradshaw, "Mechanisms of amino acid-mediated lifespan extension in Caenorhabditis elegans", BMC Genetics, 16. 8. February 2015.

[18] J. Gallinetti, E. Harputlugil, J.R. Mitchell, "Amino acid sensing in dietary-restriction-mediated longevity: roles of signal-transducing kinases GCN2 and TOR", Biochemical Journal, 449 (1). 1-10. January 2013

[19] R.C. Grandison, M.D. Piper, L. Partridge, "Amino-acid imbalance explains extension of lifespan by dietary restriction in Drosophila", Nature, 462 (7276). 1061-1064. December 2009.

[20] K. Zarse, S. Jabin, M. Ristow, "L-Theanine extends lifespan of adult Caenorhabditis elegans", European Journal of Nutrition, 51 (6). 765-768. September 2012.

[21] G. D'Antona, M. Ragni, A. Cardile, L. Tedesco, M. Dossena, F. Bruttini, F. Caliaro, G. Corsetti, R. Bottinelli, M.O. Carruba, A. Valerio, E. Nisoli, "Branched-chain amino acid supplementation promotes survival and supports cardiac and skeletal muscle mitochondrial biogenesis in middle-aged mice", Cell Metabolism, 12 (4). 362-372. October 2010.

[22] A.L. Alvers, L.K. Fishwick, M.S. Wood, D. Hu, H.S. Chung, W.A Dunn, Jr., J.P. Aris, "Autophagy and amino acid homeostasis are required for chronological longevity in Saccharomyces cerevisiae", Aging Cell, 8 (4). 353-369. August 2009.

[23] T. Stiernagle, "Maintenance of C. elegans", WormBook, (2006) 1-11. 
[24] D. Gems, D.L. Riddle, "Defining wild-type life span in Caenorhabditis elegans", The Journals of Gerontology. Series A, Biological Sciences and Medical Sciences, 55 (5). B215-219. May 2000.

[25] E.M. Vayndorf, S.S. Lee, R.H. Liu, "Whole apple extracts increase lifespan, healthspan and resistance to stress in Caenorhabditis elegans", Journal of Functional Foods, 5 (3). 1236-1243. July 2013.

[26] M.A. McCormick, J.R. Delaney, M. Tsuchiya, et. al., "A Comprehensive Analysis of replicative lifespan in 4,698 single-gene deletion strains uncovers conserved mechanisms of aging", Cell Metabolism, 22 (5). 895-906. November 2015.

[27] A. Bruckbauer, J. Banerjee, Q. Cao, X. Cui, J. Jing, L. Zha, F. Li, B. Xue, H. Shi, M.B. Zemel, "Leucine-nicotinic acid synergy stimulates AMPK/Sirt1 signaling and regulates lipid metabolism and lifespan in Caenorhabditis elegans, and hyperlipidemia and atherosclerosis in mice", American Journal of Cardiovascular Disease, 7 (2). 33-47. April 2017.

[28] X. Dong, Z. Zhou, L. Wang, B. Saremi, A. Helmbrecht, Z. Wang, J.J. Loor, "Increasing the availability of threonine, isoleucine, valine, and leucine relative to lysine while maintaining an ideal ratio of lysine:methionine alters mammary cellular metabolites, mammalian target of rapamycin signaling, and gene transcription", Journal of Dairy Science, 101(6). 5502-5514. June 2018.

[29] K. Tajiri, Y. Shimizu, "Branched-chain amino acids in liver diseases", World Journal of Gastroenterology, 19 (43). 7620-7629. November 2013.

[30] Y. Macotela, B. Emanuelli, A.M. Bang, D.O. Espinoza, J. Boucher, K. Beebe, W. Gall, C.R. Kahn, "Dietary leucine--an environmental modifier of insulin resistance acting on multiple levels of metabolism", PLoS One, 6 (6). e21187. June 2011.

[31] F.J. Dijk, M. van Dijk, S. Walrand, L.J.C. van Loon, K. van Norren, Y.C. Luiking, "Differential effects of leucine and leucineenriched whey protein on skeletal muscle protein synthesis in aged mice", Clinical Nutrition ESPEN, 24. 127-133. April 2018.

[32] J. Gao, H. Guo, Y. Sun, F. Ge, "Differential accumulation of leucine and methionine in red and green pea aphids leads to different fecundity in response to nitrogen fertilization", Pest Management Science, 74(8). 1779-1789. August 2018.

[33] D. Cristina, M. Cary, A. Lunceford, C. Clarke, C. Kenyon, "A regulated response to impaired respiration slows behavioral rates and increases lifespan in Caenorhabditis elegans", PLoS Genetics, 5 (4). e1000450. April 2009.

[34] C. Liang, B.J. Curry, P.L. Brown, M.B. Zemel, "Leucine modulates mitochondrial biogenesis and SIRT1-AMPK signaling in C2C12 myotubes", Journal of Nutrition \& Metabolism, 2014. 239750. October 2014.

[35] L. Fu, A. Bruckbauer, F. Li, Q. Cao, X. Cui, R. Wu, H. Shi, M.B. Zemel, B. Xue, "Leucine amplifies the effects of metformin on insulin sensitivity and glycemic control in diet-induced obese mice", Metabolism, 64 (7). 845-856. July 2015.

[36] A. Longchamp, T. Mirabella, A. Arduini, M.R. MacArthur, A. Das, J.H. Trevino-Villarreal, C. Hine, I. Ben-Sahra, N.H. Knudsen, L.E. Brace, J. Reynolds, P. Mejia, M. Tao, G. Sharma, R. Wang, J.M. Corpataux, J.A. Haefliger, K.H. Ahn, C.H. Lee, B.D. Manning, D.A. Sinclair, C.S. Chen, C.K. Ozaki, J.R. Mitchell, "Amino acid restriction triggers angiogenesis via GCN2/ATF4 regulation of VEGF and H2S production", Cell, 173 (1). 117-129. e14. March 2018.

[37] V. Carraro, A.C. Maurin, S. Lambert-Langlais, J. Averous, C. Chaveroux, L. Parry, C. Jousse, D. Ord, T. Ord, P. Fafournoux, A. Bruhat, "Amino acid availability controls TRB3 transcription in liver through the GCN2/eIF2 alpha/ATF4 pathway", Plos One, 5 (12). e15716. December 2010.

[38] J.M. Kingsbury, N.D. Sen, M.E. Cardenas, "Branched-chain aminotransferases control TORC1 signaling in Saccharomyces cerevisiae", PLoS Genetics, 11 (12). e1005714. December 2015.

[39] T.P. Nguyen, A.R. Frank, J.L. Jewell, "Amino acid and small GTPase regulation of mTORC1", Cellular Logistics, 7 (4). e1378794. September 2017.

[40] C. Kurino, T. Furuhashi, K. Sudoh, K. Sakamoto, "Isoamyl alcohol odor promotes longevity and stress tolerance via DAF-16 in Caenorhabditis elegans", Biochemical and Biophysical Research Communications, 485 (2). 395-399. April 2017.

\section{Supporting Information}

Table S1. Effects of amino acids other than BCAAs on the lifespan of $C$ elegans

\begin{tabular}{|c|c|c|c|c|c|c|}
\hline Amino acids & Concentration $(\times)$ & Number of worms & Median lifespan & Mean lifespan $( \pm$ SEM) & P value & Change (\%) \\
\hline \multirow{4}{*}{ Cys } & 0 & 30 & $17 \pm 0.440$ & $16.600 \pm 0.679$ & -- & \\
\hline & 0.1 & 29 & $16 \pm 0.670$ & $16.103 \pm 0.665$ & 0.604 & \\
\hline & 1 & 29 & $17 \pm 0.884$ & $16.483 \pm 0.661$ & 0.902 & \\
\hline & 10 & 29 & $14 \pm 0.535$ & $13.241 \pm 0.839$ & $0.003 * *$ & -20.23 \\
\hline \multirow{4}{*}{ Gly } & 0 & 30 & $17 \pm 0.440$ & $16.600 \pm 0.679$ & - & \\
\hline & 0.1 & 30 & $17 \pm 0.497$ & $16.533 \pm 0.531$ & 0.939 & \\
\hline & 1 & 30 & $16 \pm 0.596$ & $15.833 \pm 0.432$ & 0.345 & \\
\hline & 10 & 30 & $16 \pm 0.911$ & $15.967 \pm 0.543$ & 0.643 & \\
\hline \multirow{4}{*}{ Glu } & 0 & 30 & $17 \pm 0.440$ & $16.600 \pm 0.679$ & -- & \\
\hline & 0.1 & 30 & $16 \pm 0.685$ & $16.000 \pm 0.661$ & 0.529 & \\
\hline & 1 & 29 & $18 \pm 0.523$ & $17.655 \pm 0.458$ & 0.206 & \\
\hline & 10 & 29 & $17 \pm 0.356$ & $16.172 \pm 0.630$ & 0.647 & \\
\hline \multirow{4}{*}{ Ser } & 0 & 30 & $17 \pm 0.440$ & $16.600 \pm 0.679$ & -- & \\
\hline & 0.1 & 30 & $16 \pm 0.781$ & $16.533 \pm 0.626$ & 0.943 & \\
\hline & 1 & 30 & $19 \pm 1.086$ & $17.167 \pm 0.801$ & 0.592 & \\
\hline & 10 & 28 & $17 \pm 0.507$ & $16.714 \pm 0.711$ & 0.908 & \\
\hline \multirow{4}{*}{ Arg } & 0 & 35 & $17 \pm 0.976$ & $16.286 \pm 0.705$ & -- & \\
\hline & 0.1 & 34 & $17 \pm 0.574$ & $17.118 \pm 0.641$ & 0.387 & \\
\hline & 1 & 34 & $17 \pm 0.583$ & $17.265 \pm 0.664$ & 0.316 & \\
\hline & 10 & 33 & $18 \pm 0.480$ & $16.667 \pm 0.658$ & 0.695 & \\
\hline \multirow{4}{*}{ Ala } & 0 & 35 & $17 \pm 0.976$ & $16.286 \pm 0.705$ & -- & \\
\hline & 0.1 & 34 & $20 \pm 0.648$ & $19.265 \pm 0.858$ & $0.009<0.01 * *$ & 18.29 \\
\hline & 1 & 35 & $19 \pm 0.828$ & $17.771 \pm 0.810$ & 0.171 & \\
\hline & 10 & 35 & $15 \pm 0.680$ & $14.971 \pm 0.603$ & 0.161 & \\
\hline
\end{tabular}




\begin{tabular}{|c|c|c|c|c|c|c|}
\hline Amino acids & Concentration $(\times)$ & Number of worms & Median lifespan & Mean lifespan $( \pm$ SEM $)$ & $P$ value & Change (\%) \\
\hline \multirow{4}{*}{ Asp } & 0 & 35 & $17 \pm 0.976$ & $16.286 \pm 0.705$ & -- & \\
\hline & 0.1 & 35 & $19 \pm 0.549$ & $18.086 \pm 0.737$ & 0.082 & \\
\hline & 1 & 35 & $17 \pm 0.976$ & $15.971 \pm 0.743$ & 0.760 & \\
\hline & 10 & 34 & $14 \pm 1.158$ & $13.941 \pm 0.753$ & $0.026<0.05^{*}$ & -14.39 \\
\hline \multirow{4}{*}{ Asn } & 0 & 35 & $15 \pm 0.476$ & $14.714 \pm 0.599$ & -- & \\
\hline & 0.1 & 35 & $16 \pm 0.486$ & $14.914 \pm 0.701$ & 0.829 & \\
\hline & 1 & 34 & $15 \pm 0.486$ & $15.529 \pm 0.635$ & 0.354 & \\
\hline & 10 & 35 & $12 \pm 0.483$ & $12.143 \pm 0.457$ & $0.001<0.01^{* *}$ & -17.47 \\
\hline \multirow{4}{*}{ Gln } & 0 & 35 & $15 \pm 0.476$ & $14.714 \pm 0.599$ & -- & \\
\hline & 0.1 & 35 & $16 \pm 1.087$ & $15.200 \pm 0.835$ & 0.638 & \\
\hline & 1 & 31 & $14 \pm 0.296$ & $13.516 \pm 0.705$ & 0.197 & \\
\hline & 10 & 33 & $13 \pm 0.638$ & $14.303 \pm 0.704$ & 0.657 & \\
\hline \multirow{4}{*}{ Phe } & 0 & 35 & $15 \pm 0.476$ & $14.714 \pm 0.599$ & -- & \\
\hline & 0.1 & 33 & $14 \pm 0.858$ & $13.727 \pm 0.660$ & 0.271 & \\
\hline & 1 & 34 & $14 \pm 0.724$ & $14.088 \pm 0.652$ & 0.481 & \\
\hline & 10 & 35 & $15 \pm 0.562$ & $14.029 \pm 0.679$ & 0.452 & \\
\hline \multirow{4}{*}{ Tyr } & 0 & 35 & $15 \pm 0.476$ & $14.714 \pm 0.599$ & -- & \\
\hline & 0.1 & 34 & $15 \pm 0.483$ & $14.471 \pm 0.782$ & 0.805 & \\
\hline & 1 & 34 & $12 \pm 1.940$ & $12.588 \pm 0.723$ & $0.026<0.05^{*}$ & -14.44 \\
\hline & 10 & 35 & $14 \pm 0.884$ & $13.829 \pm 0.744$ & 0.357 & \\
\hline \multirow{4}{*}{ Try } & 0 & 34 & $18 \pm 0.724$ & $17.794 \pm 0.542$ & -- & \\
\hline & 0.1 & 35 & $17 \pm 0.411$ & $15.571 \pm 0.666$ & $0.012<0.05^{*}$ & -12.49 \\
\hline & 1 & 35 & $18 \pm 0.486$ & $16.000 \pm 0.738$ & 0.055 & \\
\hline & 10 & 35 & $18 \pm 0.915$ & $15.829 \pm 0.724$ & $0.034<0.05^{*}$ & -11.04 \\
\hline \multirow{4}{*}{ Thr } & 0 & 34 & $18 \pm 0.724$ & $17.794 \pm 0.542$ & -- & \\
\hline & 0.1 & 34 & $18 \pm 0.395$ & $16.000 \pm 0.773$ & 0.062 & \\
\hline & 1 & 35 & $16 \pm 1.449$ & $14.229 \pm 0.701$ & $0.000<0.01^{* *}$ & -20.03 \\
\hline & 10 & 31 & $15 \pm 0.835$ & $14.548 \pm 0.766$ & $0.001<0.01^{* *}$ & -18.24 \\
\hline \multirow{4}{*}{ Lys } & 0 & 34 & $18 \pm 0.724$ & $17.794 \pm 0.542$ & -- & \\
\hline & 0.1 & 34 & $17 \pm 0.582$ & $16.235 \pm 0.768$ & 0.102 & \\
\hline & 1 & 35 & $16 \pm 0.729$ & $16.029 \pm 0.676$ & $0.046<0.05^{*}$ & -9.91 \\
\hline & 10 & 31 & $18 \pm 0.574$ & $16.529 \pm 0.742$ & 0.174 & \\
\hline \multirow{4}{*}{ His } & 0 & 34 & $14 \pm 0.630$ & $14.147 \pm 0.588$ & -- & \\
\hline & 0.1 & 34 & $16 \pm 0.449$ & $16.294 \pm 0.663$ & $0.018<0.05^{*}$ & 15.17 \\
\hline & 1 & 35 & $15 \pm 0.589$ & $16.143 \pm 0.652$ & $0.027<0.05^{*}$ & 14.10 \\
\hline & 10 & 35 & $15 \pm 0.422$ & $15.714 \pm 0.563$ & 0.058 & \\
\hline \multirow{4}{*}{ Met } & 0 & 34 & $14 \pm 0.630$ & $14.147 \pm 0.588$ & -- & \\
\hline & 0.1 & 35 & $15 \pm 0.586$ & $15.229 \pm 0.717$ & 0.249 & \\
\hline & 1 & 35 & $16 \pm 0.657$ & $16.371 \pm 0.610$ & $0.011<0.05^{*}$ & 15.72 \\
\hline & 10 & 34 & $18 \pm 0.351$ & $17.121 \pm 0.661$ & $0.001<0.01^{* *}$ & 21.02 \\
\hline \multirow{4}{*}{ Pro } & 0 & 34 & $14 \pm 0.630$ & $14.147 \pm 0.588$ & -- & \\
\hline & 0.1 & 34 & $16 \pm 0.724$ & $15.971 \pm 0.613$ & $0.035<0.05^{*}$ & 12.89 \\
\hline & 1 & 33 & $15 \pm 0.861$ & $15.333 \pm 0.720$ & 0.205 & \\
\hline & 10 & 35 & $15 \pm 0.634$ & $14.829 \pm 0.552$ & 0.401 & \\
\hline
\end{tabular}

Note: $28-35$ worms were used for each experiment. Each amino acid has three concentrations $(0.1 \times, 1 \times$ and $10 \times)$.All amino acids in this table were added into $100 \mathrm{~mL}$ NGM medium by $0.0076 \mathrm{~g}$ for the concentration of $1 \times$ (around $0.5 \mathrm{mM}$ for each amino acid).

Table S2. Amino acids other than BCAAs have differential effects on lifespan of $C$ elegans in three trials

\begin{tabular}{|c|c|c|c|c|c|c|c|}
\hline & Trial 1 & Trial 2 & Trial 3 & & Trial 1 & Trial 2 & Trial 3 \\
\hline 10 Cys & $-* *$ & $-* *$ & - & 1 Tyr & $-*$ & - & - \\
\hline 10 Asn & $-* *$ & $-* *$ & $-* *$ & 0.1 Try & $-*$ & - & + \\
\hline $0.1 \mathrm{Ala}$ & $+* *$ & + & - & 10 Try & $-*$ & - & + \\
\hline $1 \mathrm{Thr}$ & $-* *$ & + & - & 1 Lys & $-*$ & + & - \\
\hline $10 \mathrm{Thr}$ & $-* *$ & $-* *$ & - & $0.1 \mathrm{His}$ & $+*$ & - & + \\
\hline $10 \mathrm{Met}$ & $+* *$ & - & + & $1 \mathrm{His}$ & $+*$ & - & + \\
\hline 0.1 Pro & $+*$ & - & + & $1 \mathrm{Met}$ & $+*$ & + & + \\
\hline 10 Asp & $-* *$ & - & $-* *$ & & & & + \\
\hline
\end{tabular}

Note: Data of Trial 1 were listed in Table S1, and data of Trial 2 and Trial 3 were not listed. 30-35 worms were used for each experiment. The - and + represent significant $\left({ }^{*} p<0.05\right.$ or $\left.{ }^{* *} p<0.01\right)$ attenuation and elongation of lifespan relative to the control, respectively. The letter $\mathrm{n}$ means no effect. Each amino acid has three concentrations $(0.1 \times, 1 \times$ and $10 \times)$.All amino acids in this table were added into $100 \mathrm{~mL}$ NGM medium by $0.0076 \mathrm{~g}$ for the concentration of $1 \times$ (around $0.5 \mathrm{mM}$ for each amino acid). 